Recepción: 19 / 07 / 2018

Aceptación: 25 / 09 / 2018

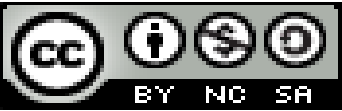

Ciencias de la educación

Publicación: 05 / 10 / 2018

Artículo de investigación

\title{
Análisis acerca de la práctica docente en un proceso innovador
}

\section{Analysis about the teaching practice in an innovative process}

\section{Análise sobre a prática docente em um processo inovador}

\author{
Luzmila M. Valverde-Medina ${ }^{\mathrm{I}}$ \\ luzmilavalverde@yahoo.es \\ Luis C. Caicedo-Perlaza II \\ luiscaicedoperlaza@yahoo.es \\ Celina Quiñonez-Quiñonez ${ }^{\text {III }}$ \\ celina_qq@gmail.com
}

Correspondencia: luzmilavalverde@yahoo.es

\footnotetext{
I Magíster en Investigación para el Desarrollo Educativo, Diplomado Superior en Práctica Docente Universitaria, Especialista en Educación Superior, Licenciada en Ciencias de la Educación Especialidad Química y Biología, Docente de la de la Universidad Técnica Luis Vargas Torres de Esmeraldas, Esmeraldas, Ecuador.

II Magíster en Investigación para el Desarrollo Educativo, Diplomado Superior en Práctica Docente Universitaria, Especialista en Educación Superior, Licenciado en Ciencias de la Educación Especialidad Química y Biología, Docente de la de la Universidad Técnica Luis Vargas Torres de Esmeraldas, Esmeraldas, Ecuador.

III Magíster en Educación Mención Educación Superior, Diploma Superior en Currículo y Didáctica, Diploma Superior en Investigación Educativa, Diploma Superior en Tecnología Gerencia y Liderazgo, Licenciado en Ciencias de la Educación, Profesor de Enseñanza Secundaria en la Especialidad de Educación Física, Docente de la de la Universidad Técnica Luis Vargas Torres de Esmeraldas, Esmeraldas, Ecuador.
} 


\title{
Resumen
}

En el presente artículo, presenta un análisis sobre el proceso de innovación de la práctica docente, considerándose como eje al aprendizaje más que a la enseñanza, desde el marco de cambios en los cánones de la educación. La reflexión se basa en primera instancia, en el proceso de innovación y luego en la adhesión de este proceso en la tarea docente para contribuir con algunas ideas de intervención que tienen como objetivo realizar cambios por desarrollarse en el aula. La metodología de la investigación se fundamenta con los métodos inductivo y deductivo, en dos momentos diferentes del estudio: cuando se obtiene los datos y cuando se procede a examinarlos e interpretarlos. Las técnicas empleadas son cualitativas, con mínimos apoyos cuantitativos; aplicándose: encuesta; observación de acciones y de textos escritos; cuestionarios y evaluaciones. Los resultados evidenciaron prácticas innovadoras en el ejercicio de la docencia, áreas de oportunidades para ser mejorada, y un proceso grupal de redimensión del aprendizaje en la comunidad.

Palabras clave: Práctica docente; innovación; aprendizaje; procesos educativos; estudiantes; enseñanza.

\begin{abstract}
$\mathrm{n}$ the present article, it presents an analysis about the innovation process of the teaching practice, considering as an axis the learning more than the teaching, from the framework of changes in the canons of education. The reflection is based on the first instance, on the process of innovation and then on the adhesion of this process in the teaching task to contribute with some ideas of intervention that aim to make changes to be developed in the classroom. The methodology of the investigation is based on the inductive and deductive methods, in two different moments of the study: when the data is obtained and when it is proceeded to examine and interpret them. The techniques used are qualitative, with minimal quantitative support; applied: survey; observation of actions and written texts; questionnaires and evaluations. The results showed innovative practices in the teaching practice, areas of opportunities to be improved, and a group process of redimensión of learning in the community.
\end{abstract}

Keywords: Teaching practice; innovation; learning; educational processes; students; teaching. 


\section{Resumo}

No presente artigo, apresenta uma análise sobre o processo de inovação da prática docente, considerando como eixo o aprendizado mais do que o ensino, a partir do quadro de mudanças nos cânones da educação. A reflexão baseia-se, em primeira instância, no processo de inovação e, em seguida, na adesão deste processo na tarefa de ensino de contribuir com algumas ideias de intervenção que visam fazer com que as mudanças sejam desenvolvidas em sala de aula. A metodologia da investigação baseia-se nos métodos indutivo e dedutivo, em dois momentos distintos do estudo: quando os dados são obtidos e quando se procede a examiná-los e interpretálos. As técnicas utilizadas são qualitativas, com suporte quantitativo mínimo; aplicado: pesquisa; observação de ações e textos escritos; questionários e avaliações. Os resultados mostraram práticas inovadoras na prática docente, áreas de oportunidades a serem melhoradas e um processo grupal de redimensión de aprendizagem na comunidade.

Palavras chave: Prática de ensino; inovação; aprendizagem processos educativos; estudantes; ensinando.

\section{Introducción}

Actualmente existe una necesidad latente en cuanto a la eficiencia y eficacia para el docente en el momento de impartir su cátedra, por tanto, es un requisito la innovación en la práctica docente que el profesor presenta. Esto conlleva a desarrollar y evaluar de manera constante, las competencias básicas que le permitirán al docente introducir y aplicar diferentes herramientas en el proceso enseñanza-aprendizaje.

Es de total relevancia el tema de la innovación en la práctica docente, puesto que al innovar dentro de la práctica pedagógica por medio de la aplicación de diversas técnicas de enseñanza, el estudiante logrará asimilar rápidamente los contenidos y alcanzará un aprendizaje significativo donde sabrá reflexionar, y analizar con carácter crítico bajo el enfoque por competencias, así mismo podrá relacionar los hechos de la actualidad con los temas, obteniendo una completa transversalidad.

Se presenta un análisis en torno al proceso que recorre la innovación de la práctica docente en instituciones de educación superior. La innovación educativa es un proceso que consiste en 
incorporar algo nuevo, se puede caracterizar como un cambio creativo y duradero en cualquier nivel de las prácticas educativas, que se realiza de manera intencional, que produce modificaciones profundas en el sistema de generación y transferencia de conocimientos, habilidades, actitudes y valores, con la articulación de la participación de los agentes y que mejora la calidad de algún aspecto significativo del hecho educativo. (Silva S., 2015)

\section{Desarrollo}

El planteamiento de innovaciones educativas dentro de un proceso de aprendizaje por competencias se ha planteado por tanto como un procedimiento sistemático e intencionado que solicita conocimiento y uso de diferentes técnicas y estrategias para efectuarlas. La mejora al utilizar la innovación dentro de la práctica docente es reducir el esfuerzo; aumentar la rapidez en obtener resultados; aumentar la calidad y satisfacer nuevas demandas.

Los conceptos, creatividad e innovación tienen una vinculación de importancia ya que la creatividad ha sido identificada como una condición básica para generar innovación y cambio, un punto de partida preferentemente vinculada a las características personales de los individuos.

Existe un relativo consenso respecto a definir la creatividad como una capacidad o potencial humano para generar ideas nuevas, dentro de un marco de valores y comunicarlas (De la Torre, 2003).

La innovación se entiende como un componente de cambio de cualquier proceso de desarrollo. En el campo educativo, la innovación se refiere a tres espacios a saber "en la estructura burocrática, en los centros de trabajo escolares y en el espacio específico del aula" (Uc et al, 2009). Esta última se manifiesta en la introducción de cambios en el trabajo del docente. La educación juega en la globalización un papel protagonista. Sostiene un compromiso importante como interventora en el progreso social y económico a través de sus programas educativos y los principios sobre los cuales prepara a los estudiantes que, a su vez, serán los actores del desarrollo. La innovación en la educación se torna en un componente que dota de dinamismo a los procesos administrativos y las relaciones humanas en sus diferentes espacios. Las instituciones como sistemas formalizados responden a las demandas de los Estados, siendo las proveedoras de la oferta educativa que nutre de recursos humanos calificados para el desarrollo de la actividad económica y social. La formación para el trabajo y los mercados laborales se convierte en el 
punto de partida para la actividad educativa. Esta formación es cada vez más compleja y exigente en conocimientos, en el manejo de la información, en el desarrollo de competencias, de habilidades y capacidades. Si bien la actividad profesional se torna más amplia, también se vuelve menos repetitiva y más creativa. La educación centrada en el aprendizaje más que en la enseñanza y en los procesos educativos centrados en el estudiante (MUM, 2007) entre otras políticas, derivaron de los criterios para responder a las necesidades del mundo contemporáneo de acuerdo con la Declaración de Bolonia de 1999 (Docampo, 2001), con la Organización para la Cooperación y el Desarrollo Económico (OCDE) y la Oficina Estadística de la Unión Europea (Eurostat) (Calderón et al, 2009), la Organización de las Naciones Unidas para la Educación, la Ciencia y la Cultura (UNESCO), el Banco Mundial y la Asociación Nacional de Universidades e Instituciones de Educación Superior (ANUIES) (LEI, 2009).

En este contexto, las instituciones de educación superior se ven en la necesidad ofrecer la currícula que facilite a sus egresados la participación en la globalización, y quienes ayudan en gran parte en la motivación de esta participación profesional son, sin duda, los docentes. El perfil de egreso de las diferentes carreras representa la conexión entre las Instituciones de educación superior (IES) y las actividades económicas y sociales.

Los docentes de estas IES tienen la responsabilidad de llevar a sus alumnos al perfil de egreso. El objetivo de llegar al perfil de egreso se encuentra centrado en la tarea de los docentes y alumnos en práctica dentro y fuera de las aulas. La innovación de la práctica docente en este contexto consiste en poner en acción el cambio de paradigma de la educación contemporánea, establecido en centrar el trabajo del docente en el aprendizaje.

El cambio, requiere más que un curso de capacitación. Es de considerar que el acto de la práctica docente tiene lugar en las instituciones y, por lo tanto, que su ejercicio también se ve acotado por la filosofía, los principios, las políticas y las normas que la institución marca como parte de una visión sobre el quehacer teleológico de educar que incluye, entre otros aspectos, una "reflexión en torno al modelo de ciudadano que se quiere potenciar, ... la construcción social de valores como la autonomía, la responsabilidad o la cooperación a través de los procesos educativos". (Palomero (2009:17) 


\section{Innovación y cambio}

Ahora bien, no se trata sólo de cambiar, sino de innovar. Al respecto es primordial establecer la diferencia entre cambio e innovación. Por una parte, el cambio se relaciona con cualquier práctica que es una novedad para las personas que la realizan, esta práctica podría producirse al azar y no tener efectos significativos; por ejemplo, no porque haya un cambio éste garantiza la mejora. Por otra parte, la innovación es el "esfuerzo deliberado por mejorar la práctica en relación con ciertos objetivos que se desean.” (Antúnez, 1998, p. 6)

La innovación en la formación pedagógica a la que se alude en este texto, es toda acción planificada para modificar la forma de pensar y de actuar de los docentes en formación, teniendo como propósito el aprendizaje de sus estudiantes por medio de la implementación de novedades en cualquier aspecto del currículo, con miras al desarrollo de las competencias para la vida, entre ellas, para el aprendizaje permanente; pues en la educación normal, se educa para la utopía, es decir, para el lugar que aún no existe y sin embargo, es posible construir, que en palabras de Datri (2014) significa un mundo bello. De esta forma, las innovaciones son el efecto de los cambios planificados, otorgándole al educador un talante disidente, de no conformación con el statu quo y, en consecuencia, proclamarse como un promotor del cambio y de la innovación en su práctica pedagógica.

\section{Creatividad}

A razón de que el maestro es el recurso humano para promover las innovaciones en el proceso educativo, éste puede ser también la fuente principal de la resistencia al cambio, por tanto, es pertinente mencionar a la creatividad, que se debe fomentar para que se favorezca el espíritu creativo e innovador en la formación pedagógica, iniciando en el pensamiento para después concretarse en la acción educativa.

"El pensamiento creativo está orientado hacia el futuro, intenta encontrar un modo de hacer las cosas mejor que las anteriores" (Solá, 2011, p. 78), a fin de transformar el mundo, generando nuevas ideas y significados. En este sentido, la innovación en el ámbito educativo no necesariamente significa inventar algo nuevo, sino que se relaciona con la creatividad del docente cuando halla formas con las cuales puede mejorar su práctica. A tenor de esta idea, cabe retomar el concepto de trascendencia anteriormente mencionado, puesto que un docente creativo va más 
allá, encuentra la forma de modificar su entorno y de adaptar todo aquello que tiene disponible en beneficio del proceso de enseñanza y de aprendizaje.

\section{La profesionalización docente}

Para Medina Rivilla (2015) la innovación "facilita resolver algunos de los problemas presentes o limita la influencia de condiciones adversas para el desarrollo y desenvolvimiento del sistema." (p. 109) Por consiguiente, la innovación se convierte en un factor fundamental, que se plantea como una respuesta ante los retos que representa educar en tiempos adversos, en los cuales la posmodernidad causa estragos, haciendo que en los valores permee el relativismo, que el impacto de la tecnología conlleve a la superación de múltiples límites y soslaye a la intersubjetividad, así como en la "oposición al mundo de la vida, del sistema como elemento capaz de plantear una ciencia social y del hombre, aunque para ello se tenga que renunciar al propio hombre y en definitiva, al humanismo" (Colom, 1997, p. 9), por tal motivo, el normalista como agente de cambio social, tendrá la oportunidad de incidir en la realidad, desarrollando su práctica educativa a través de propuestas lúcidas, pertinentes e innovadoras, como parte de su obligación moral.

De hecho, en esta época signada por las implicaciones de la reforma educativa que está por arribar en Educación Básica, también se está perfilando un cambio en la formación inicial docente, espacio donde se aprende a enseñar, pero también a analizar la práctica, proceso que para Zabala (2000) es inseparable de la innovación, "ya que sólo podemos innovar a partir de la detección de las dificultades o carencias de lo que queremos cambiar.” (p. 233). Precisamente en ese espacio, ya existen las condiciones para la reforma, puesto que el análisis y la reflexión de la práctica que actualmente se promueven, hacen fértil el terreno para proponer la innovación, la cual, según Colom, significa “crear, descubrir, ampliar en definitiva el saber” (1997, p. 15).

En efecto, "se trata más bien de formar para la innovación a personas capaces de evolucionar, de adaptarse a un mundo en rápida mutación y de dominar el cambio” (Delors, 1996, p. 37). Cabe afirmar que la innovación es el nivel más alto de la competencia, dado que implica la actuación autónoma, en conjunción con la creatividad, pues el desempeño se podrá dar en diversos contextos, teniendo en cuenta que el valor inherente del docente, como ser racional y libre, se representa a través de su dignidad. En consecuencia, la idea de grandeza (dignitas) se fortalece por medio de la capacidad innovadora del docente, no sólo como un derecho, sino también como 
un deber, que se comienza a fraguar en el Modelo Educativo 2016 para la Educación Básica, contemplando también a la formación inicial, en la cual se tiene como objetivo que "los docentes construyan interacciones educativas significativas con creatividad e innovación, con el fin de estimular a sus alumnos a alcanzar los resultados esperados" (SEP, 2016, p. 16-17), dentro de una perspectiva humanista, que se basa en desarrollar el potencial de las personas para que puedan participar de forma activa y responsable en la sociedad.

La innovación siempre tenderá hacia el humanismo puesto que la finalidad de la evolución es mejorar la sociedad, a partir del trabajo en el campo educativo, donde innovar implica realizar cambios en los múltiples aspectos de la teoría y práctica curricular (métodos, metodologías, recursos y materiales, formas de organización, de gestión, de convivencia, etc.), de ahí que la innovación surja desde el entramado teórico en conjunción con la praxis, siempre y cuando el docente apele a la alteración del orden, para mostrar otros mundos posibles, de tal manera que "una práctica reflexiva aumente la capacidad de innovación” (Perrenoud, 2011, p. 46). Entonces, el humanismo como perspectiva, recurre al cultivo de la reflexión para desarrollar el pensamiento creativo y, en definitiva, la capacidad de innovación.

La profesionalización docente es en sí misma una estrategia para la implementación de innovaciones, ya que requiere de los profesores construcción de aprendizajes tanto del contenido de las innovaciones que se pretenden implementar, así como del procedimiento para su puesta en práctica.

Según Grande y Pemoff (2002), se debe considerar tres criterios para los procesos de formación profesional:

- La formación debe considerarse como un proceso de construcción de una cultura profesional.

- La formación sólo será eficaz si el conocimiento esté vinculado al conocimiento práctico.

- Las instituciones escolares deben ser los núcleos de la formación para el desarrollo profesional".

Rivas, 2004, menciona que: 
"El gran reto en la profesionalización docente conlleva al enriquecimiento del propio pensamiento pedagógico del docente y el mejoramiento de las competencias didácticas, asi como de los principios pedagógiccos que debe poseer el maestro para el bien de sus alumnos y favoreciendo el proceso enseñanza-aprendizaje mismo que será eficiente si se trabaja de forma organizada y compartida"

La Asociación Nacional de Universidades e Instituciones de Educación Superior (ANUIES, 2003) ha definido cinco ámbitos de innovación:

- Planes y programas de estudio,

- proceso educativo,

- uso de tecnologías de información y comunicación,

- modalidades alternativas para el aprendizaje y

- gobierno, dirección y gestión.

Una innovación educativa efectiva es aquella que logra integrarse con otros componentes del proceso educativo o pedagógico, con los cuales necesariamente interactúa y se complementa para lograr frentes más amplios de mejoramiento de la calidad, de la cobertura y de la eficiencia (Blanco y Messina, 2000).

El cambio de la innovación en la práctica del docente, es un paradigma, por lo tanto, dicho acontecimiento debe estar enfocado en la formación de competencias acorde a la reestructuración de las sociedades contemporáneas occidentales (Moreno, 2009).

Estudios recientes como los de Almerich, 2005, coinciden en señalar la necesidad de formación de competencias orientadas a la innovación en los docentes como un medio para cambiar sus actitudes hacia los procesos de cambio y mejora en las instituciones.

\section{Conclusiones}

En cuanto a la práctica de la docencia, permite ser caracterizarla como no tradicional, con prácticas innovadoras, desempeñada con competencia profesional. Sin embargo, se debe equilibrar más las acciones que cubran de igual manera con los tres objetivos o variables seleccionadas para caracterizar el aprendizaje significativo. 
Es necesario que los procesos asumidos por las y los docentes innovadores, en las instituciones se crearan espacios de reflexión donde se promoviera el diálogo entre los docentes para compartir, analizar, criticar y promover los nuevos modelos educativos. Ya que como dice Mialaret (1976, citado por Arredondo et al., 1997), la docencia, como la educación, puede ser asumida como sistema, como producto y como proceso. Otro resultado que se vincula con la demanda de coherencia en el quehacer institucional, es cómo vincular las innovaciones centradas en los procesos, cuyas constataciones van a ser cualitativas, con la demanda de reportar calificaciones numéricas que, por consiguiente, está fijando su punto de mira en los productos obtenidos, a partir de ciertos insumos. Ello presenta otra área de oportunidad a mejorar en el ejercicio profesional de la docencia: el diseño de rúbricas que cumplan con el propósito de cuantificar de manera reflexiva y crítica, lo cualificado. Hasta ahora, creo que lo he hecho de una manera un tanto arbitrario. Finalmente, es importante mencionar a otro de los elementos estructurantes de las dimensiones del proceso educativo: la información.

\section{Referencias bibliográficas}

Almerich, G., J. M. Suárez, N. Orellana, C. Belloch, R. BoeI. Gastaldo. (2005). Diferencias en los conocimientos de los recursos tecnológicos en profesores. Revista RELIEVE, vol. 11, núm. 2, pp. 127-146.

Andueza, María. (1997). Dinámicas de grupos en Educación. México: Trillas.

Arredondo, Miguel et al. (2000). Notas para un modelo de docencia. En Arredondo, Miguel. \& Díaz-Barriga, Ángel (Comp.), Formación pedagógica de profesores Universitarios. Teorías y experiencias en México. México: UNAM-CESU.

ANUIES (2003). Documento estratégico para la innovación en la educación superior. México, ANUIES.

Bazdresh, José Miguel. (2000). Práctica Educativa. En Trasformar la práctica. Jalisco: Educar.

Blanco R. y G. Messina. (2000). Estado del arte sobre las innovaciones educativas en América Latina. Colombia, Convenio Andrés Bello, UNESCO. 
Bogdan, Robert, Taylor, Steven. (1987). Métodos cualitativos de investigación. Barcelona: Paidós

Borell, Francese. (2001). Cómo trabajar en equipo y crear relaciones de calidad con jefes y compañeros. Barcelona: Gestión 2000.

Cámara, Gabriel. (Coord.). (2006). Enseñar y aprender con interés. México: Siglo XXI.

Cánovas-Marmo, Célica. (1996). Un modelo parta la formación del pensamiento histórico en estudiantes universitarios. Tesis de Maestría. México: Universidad Iberoamericana. León.

Cañal, Pedro (Coord.) (2002). La innovación educativa. Madrid. Universidad Internacional de Andalucía: AKAL

Castells, Manuel et al. (1994). Nuevas perspectivas críticas en la educación. Barcelona: Paidós Educador.

Coll, César et al. (2000). El constructivismo en el aula. Barcelona: Graó.

Croll, Paul. (1995). La observación sistemática en el aula. Madrid: Editorial La Muralla.

De La Garza, Enrique. (2000). Seminario de metodología cualitativa. México: Universidad Autónoma de Aguascalientes.

De la Torre, S. (2003). Dialogando con la creatividad. (1ª Ed). Barcelona: Octaedro.

Delors, Jacques. (1996). La educación encierra un tesoro. Informe de la Comisión Internacional sobre la educación para el siglo XXI. UNESCO.

Dijk, Van Teun. (2000). El discurso como interacción social. Tomo II. Barcelona: Gedisa.

Giraux, Henry A. (2002). Los profesores como intelectuales. Hacia una pedagogía crítica del aprendizaje. Barcelona: Paidós.

Goetz, Judith \& LeCompte, Margaret. (1998). Etnografía y diseño cualitativo en investigación educativa. Madrid: Morata.

Freire, Pablo. (1997). Pedagogía de la autonomía. México: Siglo XXI.

Giroux, Henry. (1998). Los profesores como intelectuales. Barcelona: Pailón-M.E.C. 
González, María Teresa \& Escudero, Juan Manuel. (1990). Innovación educativa: teorías y procesos de desarrollo. Barcelona: Humanitas.

Grande, B., Pemoff, J (2002). Innovación Educativa Institucional. (1 ${ }^{a}$. Ed). Argentina: Magisterio del Río de la Plata.

Hamel, Jacques, Dufur, Sephane \& Fortin, Dominic. (1991). Case Methods Qualitative Research Methods. London: Publication New Park.

Latapí, Pablo. (1998). Los cuatro pilares de la Educación. (Artículo, fotocopia s/r)

Moreno, T. (2009). Competencias en educación superior: un alto en el camino para revisar la ruta de viaje. Revista en Perfiles Educativos, vol. 31, núm. México: IISUE-UNAM,

Patterson, Leslie et al. (2002). Los maestros son investigadores. Reflexiones y acciones. México: Trillas.

Ramírez, M.E., Torres, JL., Suárez, L., Ortega, P. (2006). Vínculos entre la investigación y la práctica en la matemática escolar. Seminario Repensar las Matemáticas, una innovación en la formación docente.

Rivas, M. (2004). Innovación educativa. (1ª Ed). Madrid: Síntesis.

Schön, Donald. (1987). La formación de profesores reflexivos. España: Paidós-Ministerio de Educación y Ciencia

Stake, Robert. (1999). Investigación con estudio de casos. Madrid: Morata

Wertsch, James V. (1990). Vygotsky y la formación social de la mente. Cognición y desarrollo humano. Buenos Aires: Paidós.

Zabalza, Miguel. (2006). Competencias docentes del profesorado universitario. Madrid: Narcea. Zemelman, Hugo. (1992). Dialéctica y apreciación del presente. Las funciones de la totalidad. En Usos críticos de la razón. Uso crítico de la razón. México: Colegio de México y Anthropos. 